\title{
Hydromagnetic boundary layer flow of a dusty fluid in a porous medium over a stretching sheet with slip effect
}

\author{
Noorzehan Fazahiyah Md Shab a, Anati Ali b, ${ }^{*}$ \\ a Department of Mathematics and Statistics, Faculty of Science, Technology and Human Development, Universiti Tun Hussein Onn Malaysia, \\ 86400 Parit Raja, Johor, Malaysia \\ b Department of Mathematics, Faculty of Science, Universiti Teknologi Malaysia, 81310 UTM Johor Bahru, Johor, Malaysia
}

* Corresponding author: anati@utm.my

\section{Article history}

Received 19 February 2017

Accepted 20 July 2017

\begin{abstract}
This paper investigated the problem of hydromagnetic boundary layer flow and heat transfer of a dusty fluid over a stretching sheet through a porous medium. The velocity slip was considered instead of the no-slip condition at the boundary. The governing partial equations were reduced into a set of non-linear ordinary differential equations by using the suitable similarity transformation. The transformed equations were numerically integrated using bvp4c in Matlab. The effects of various physical parameters on the velocity and temperature profiles of both phases, such as fluid-particle interaction parameter, magnetic parameter, mass concentration parameter, porosity parameter and Prandt number were obtained and analyzed through several plots. Useful discussions were carried out with the help of plotted graphs and tables. Under the limiting cases, the obtained numerical results were compared and found to be in good agreement with previously published results.
\end{abstract}

Keywords: Boundary layer flow, dusty fluid, hydromagnetic flow

\section{INTRODUCTION}

Boundary layer flow and heat transfer of a fluid over a stretching surface has attracted many researchers in the last few decades. Since then, it has a wide range of applications in various fields such as polymer processing industries, extrusion, wire drawing, metal spinning, and hot rolling (Fischer, 1976). Crane (1970) pioneered the study of stretching sheet.. He presented an exact analytical solution for the steady two-dimensional stretching of a plate in a quiescent fluid. Then, Grubka and Bobba (1985) investigated heat transfer characteristics of a continuous stretching surface with variable temperature. Many authors have considered various aspects of this problem ever since, namely Fazlina et al. (2010)and Mansur and Ishak (2013).

Furthermore, the investigation on two-phase boundary layer flow in which solid spherical particles are distributed in fluid becomes extensive research in fluid dynamics due to its importance in engineering applications and technical problems such as flow through packed beds, sedimentation, environmental pollution, centrifugal separation of particles and blood rheology. The first research on boundary layer of dusty gas was carried out by Chakrabarti (1977). Then, Vajravelu and Nayfeh (1992) studied the boundary layer flow of dusty fluid over a stretching surface with the presence of suction. Gireesha et al. (2011) had studied the boundary layer flow of dusty fluid and heat transfer over a stretching sheet. Vajravelu et al. (2013) studied the hydromagnetic fluid flow of a dusty fluid and heat transfer at a stretching sheet with fluid-particle suspension. The analysis showed that the transverse velocity decreased with the increasing fluidparticle interaction parameter in the presence of magnetic field.
Recently,Sharena et al. (2016) investigated the magnetohydrodynamic flow of dusty fluid past a vertical stretching sheet with Hall effect.

Motivated by previous studies, the hydromagnetic boundary layer flow of a dusty fluid in a porous medium over a stretching sheet with slip effect is investigated. We consider the flow through porous medium since it plays an important role in many applications, such as geothermal operations, petroleum industries, thermal insulation, and many more. We also consider the slip condition since the velocity slip could possibly happen over stretching sheet in dusty fluid. For example, in emulsifier and polymer processing applications. The coupled nonlinear partial differential equations governing the problem are transformed into a coupled nonlinear ordinary differential equations by using appropriate similarity transformation. These resulting nonlinear ordinary differential equations are solved numerically by bvp4c function solver in Matlab software.

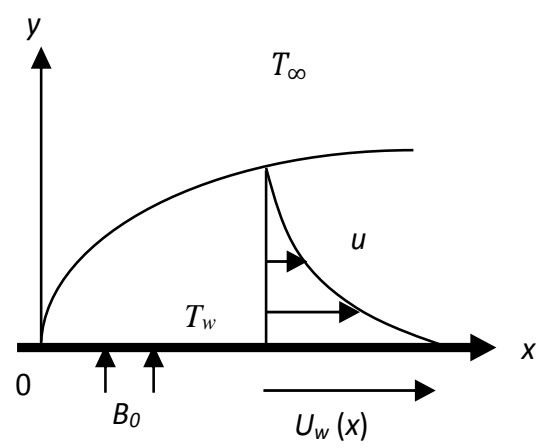

Fig. 1 Physical diagram for the problem 


\section{PROBLEM FORMULATION}

Consider a steady two-dimensional laminar boundary layer flow and heat transfer of an incompressible, electrically conducting dusty fluid over a stretching sheet through a porous medium with the presence of applied strong magnetic field. The sheet is being stretched with the velocity $U_{w}(x)$ along the $x$-axis, keeping the origin fixed.(see Fig. 1). The fluid and dust particle clouds are supposed to be static at the beginning. The non-reactive solid particles are assumed to be spherical and uniform in size. Furthermore, the density of the dust particle is taken as a constant throughout the flow.

Under these assumptions along with the boundary layer approximations, the governing boundary layer equations of both fluid and particle phase are given as

$\frac{\partial u}{\partial x}+\frac{\partial v}{\partial y}=0$

$\rho\left(u \frac{\partial u}{\partial x}+v \frac{\partial u}{\partial y}\right)=\mu \frac{\partial^{2} u}{\partial y^{2}}+\frac{\rho_{p}}{\tau}\left(u_{p}-u\right)-\sigma_{0} B_{0}^{2} u+\frac{\mu}{k_{p}} u$

$\frac{\partial}{\partial x}\left(u_{p}\right)+\frac{\partial}{\partial y}\left(v_{p}\right)=0$

$u_{p} \frac{\partial u_{p}}{\partial x}+v_{p} \frac{\partial u_{p}}{\partial y}=\frac{1}{\tau}\left(u-u_{p}\right)$

$\rho c_{p}\left(u \frac{\partial T}{\partial x}+v \frac{\partial T}{\partial y}\right)=k \frac{\partial^{2} T}{\partial y^{2}}+\frac{c_{s} \rho_{p}}{\gamma_{T}}\left(T_{p}-T\right)$

$\rho_{p} c_{s}\left(u_{p} \frac{\partial T_{p}}{\partial x}+v_{p} \frac{\partial T_{p}}{\partial y}\right)=-\frac{c_{s} \rho_{p}}{\gamma_{T}}\left(T_{p}-T\right)$

where $(u, v)$ and $\left(u_{p}, v_{p}\right)$ are the velocity components of the fluid and the dust particle phases along $x$ and $y$ directions respectively. $\mu, \rho_{\infty}$ and $\rho_{p}$ are the dynamic viscosity of the fluid, density of the fluid, and mass of dust particles per unit volume of the fluid, $T$ and $T_{p}$ are the temperature of the fluid and temperature of the dust particle, $k$ is the thermal conductivity, $c_{p}$ and $c_{s}$ are specific heat of fluid and dust particles, $\sigma$ is the electrical conductivity and $B_{0}$ is the uniform magnetic field. $\gamma_{T}$ is the temperature relaxation time whereas $\tau$ is the thermal conductivity. In deriving these equations, the drag force is considered for the interaction between the fluid and dust phases. The boundary conditions are

$$
\begin{gathered}
u=U_{w}(x)+L \frac{\partial u}{\partial y}, \quad v=0, \quad T=T_{w} \text { at } y=0 \\
u \rightarrow 0, u_{p} \rightarrow 0, \quad v_{p} \rightarrow v, T \rightarrow T_{\infty}, T_{p} \rightarrow T_{\infty}, \\
\text { as } y \rightarrow \infty
\end{gathered}
$$

where $U_{w}(x)=b x$ is the stretching sheet velocity, with prescribed surface temperature $T_{w}-T_{\infty}=A\left(\frac{x}{l}\right)$, with $b>0$ is the stretching rate, $l$ is the characteristic length and $A$ are constants.

The governing equations (1) - (6) subjected to the boundary conditions (7) can be expressed in a simpler form by introducing the following dimensionless coordinates in term of similarity variable and similarity function as

$u=b x f^{\prime}(\eta), \quad v=-\sqrt{v b} f(\eta), \quad \eta=\sqrt{\frac{b}{v}} y$

$u_{p}=b x F^{\prime}(\eta), \quad v_{p}=-\sqrt{v b} F(\eta)$

$\theta(\eta)=\frac{T-T_{\infty}}{T_{w}-T_{\infty}}, \quad \theta_{p}(\eta)=\frac{T_{p}-T_{\infty}}{T_{w}-T_{\infty}}$.

where $v$ is the kinematic viscosity of the fluid.
By using the similarity equations from (8), we obtain the following nonlinear ordinary differential equations:

$f^{\prime 2}-f f^{\prime \prime}=f^{\prime \prime \prime}+\beta \rho_{r}\left(F^{\prime}-f^{\prime}\right)-M f^{\prime}-k f^{\prime}$

$F^{\prime 2}-F F^{\prime \prime}=\beta\left(f^{\prime}-F^{\prime}\right)$

$2 f^{\prime} \theta-f \theta^{\prime}=\frac{1}{P r} \theta^{\prime \prime}+\frac{2}{3} \frac{\beta \rho_{r}}{P r}\left(\theta_{p}-\theta\right)$

$F^{\prime} \theta_{p}-F \theta_{p}^{\prime}=-\frac{2}{3} \frac{\beta}{\gamma P r}\left(\theta_{p}-\theta\right)$

where $\rho_{r}=\rho_{p} / \rho_{\infty}$ is relative density, $\beta=1 / c \tau$ is the fluid particle interaction parameter, $M=\sigma B_{0}^{2} / \rho_{\infty} c$ is the magnetic parameter, $k$ is the porosity parameter, $\operatorname{Pr}=v / \alpha$ is Prandtl number and $\gamma=c_{p} / c_{s}$ is the ratio of specific heat capacity of the fluid dan dust phase.

The boundary condition in (7) becomes

$f^{\prime}(\eta)=1+A f^{\prime \prime}(\eta), f(\eta)=0, \theta(\eta)=0 \quad$ at $\quad \eta=0$

$f^{\prime}(\eta) \rightarrow 0, \quad f(\eta) \rightarrow F(\eta), \quad F^{\prime}(\eta) \rightarrow 0$,

$\theta(\eta) \rightarrow 0, \quad \theta_{p}(\eta) \rightarrow 0 \quad$ as $\quad \eta \rightarrow 0$

where $A=L\left(\frac{b}{v}\right)^{1 / 2}$ is the slip parameter.

\section{RESULTS AND DISCUSSION}

The set of ordinary differential equations (9) - (12) subjected to boundary conditions (13) were solved numerically using the bvp4c function in MATLAB. The function is used to solve the equations due to its effectiveness in solving the boundary value problems (Mansur $e t$ $a l ., 2015)$. The numerical solutions were obtained to study the effects of various physical parameters such as fluid particle interaction parameter $\beta$, magnetic parameter $M$, porosity parameter $k$, Prandtl number $\operatorname{Pr}$ and mass concentration $\rho_{r}$ on velocity, temperature as well as the gradients of both velocity and temperature. In order to verify the accuracy of this study, Table 1 is drawn to compare the results obtained by this study with previous studies done by Grubka and Bobba (1985) and Sharena et al. (2016). The table shows excellent agreement between the three sets of results.

Table 1 Results validation for values of $-\theta^{\prime}(0)$ for existing solutions for $M=\beta=\rho_{r}=k=0$.

\begin{tabular}{cccc}
\hline Pr & $\begin{array}{c}\text { Grubka \& } \\
\text { Bobba (1985) }\end{array}$ & $\begin{array}{c}\text { Sharena et } \\
\text { al. (2016) }\end{array}$ & $\begin{array}{c}\text { Present } \\
\text { study }\end{array}$ \\
\hline 0.72 & 1.0885 & 1.0886 & 1.0886 \\
1 & 1.3333 & 1.3333 & 1.3333 \\
3 & 2.5097 & 2.5097 & 2.5097 \\
10 & 4.7969 & 4.7969 & 4.7969 \\
\hline
\end{tabular}

Figs. 2-10 depict the distributions of velocity and temperature for different values of $M, \beta, \rho_{r}, \operatorname{Pr}$ and $k$. In Fig. 2, it is shown that the increasing $M$ decreases the velocity for both fluid and dust. When the velocity of stretching sheet is high, the increase of $M$ causes the main stream velocity to reduce the velocity of the fluid and dust. Hence, as $M$ increases, the drag force which opposes the flow also increases and thus decreases the velocity of the flow.Fig. 3 shows the effect of magnetic parameter $M$ on temperature, where the temperature increases as $M$ increases. 


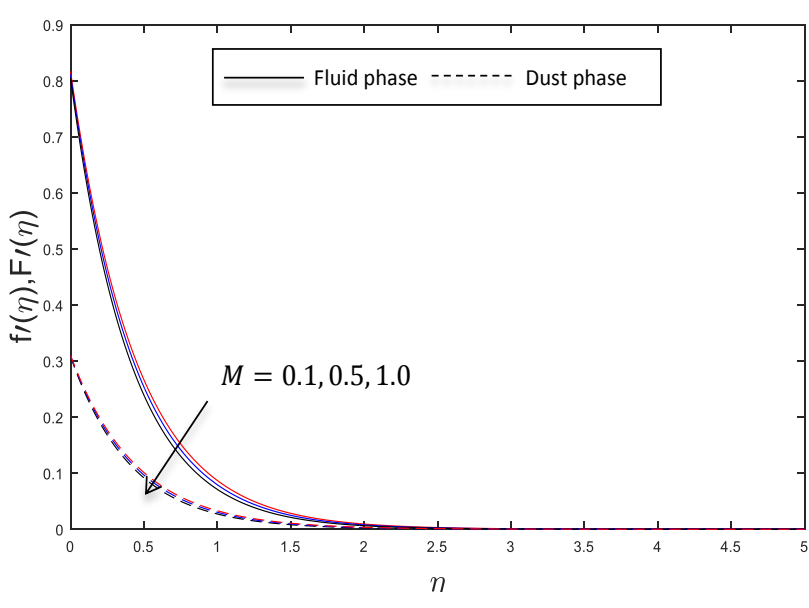

Fig. 2 Velocity profiles for fluid phase $f^{\prime}(\eta)$ and dust phase $F^{\prime}(\eta)$ for different values of $M$.

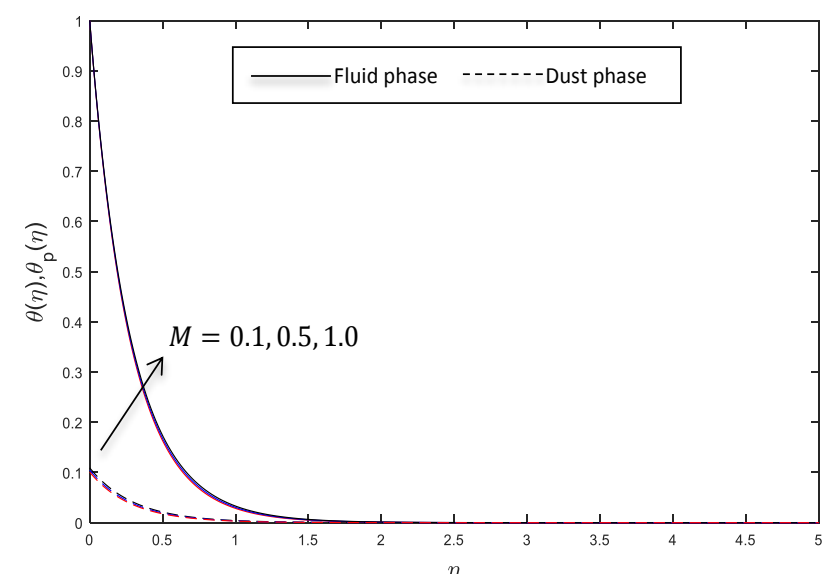

Fig. 3 Temperature profiles for fluid phase $\theta(\eta)$ and dust phase $\theta_{p}(\eta)$ for different values of $M$.

The influence of $\beta$ on velocity and temperature are shown in Figs. 4 and 5 , respectively. As $\beta$ increases, velocity of dust also increases. However, the increment of $\beta$ causes the velocity of fluid to decrease. It is expected that for a large value of $\beta$ the velocities for both fluid and dust will be the same due to the decreasing relaxation time. On the other hand, the temperature for fluid decreases and increases for dust as $\beta$ increases.

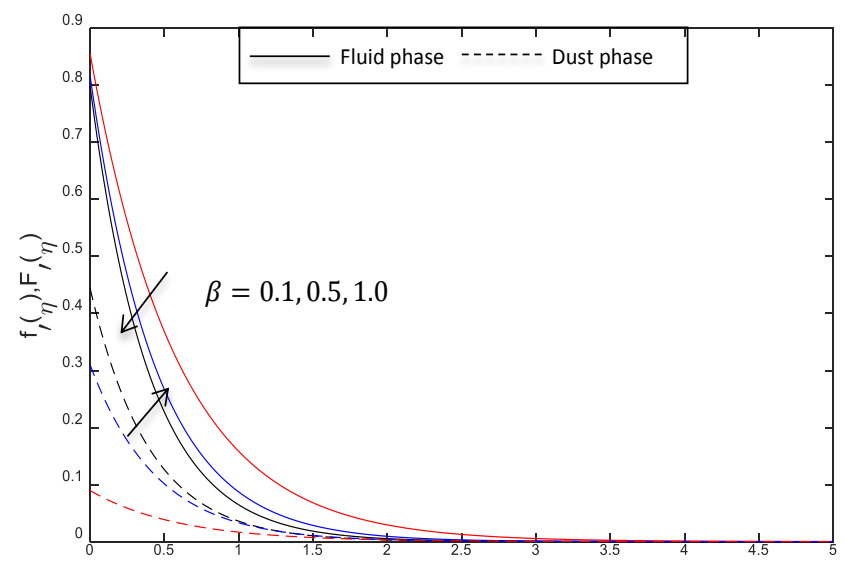

Fig. 4 Velocity profiles for fluid phase $f^{\prime}(\eta)$ and dust phase $F^{\prime}(\eta)$ for different values of $\beta$.

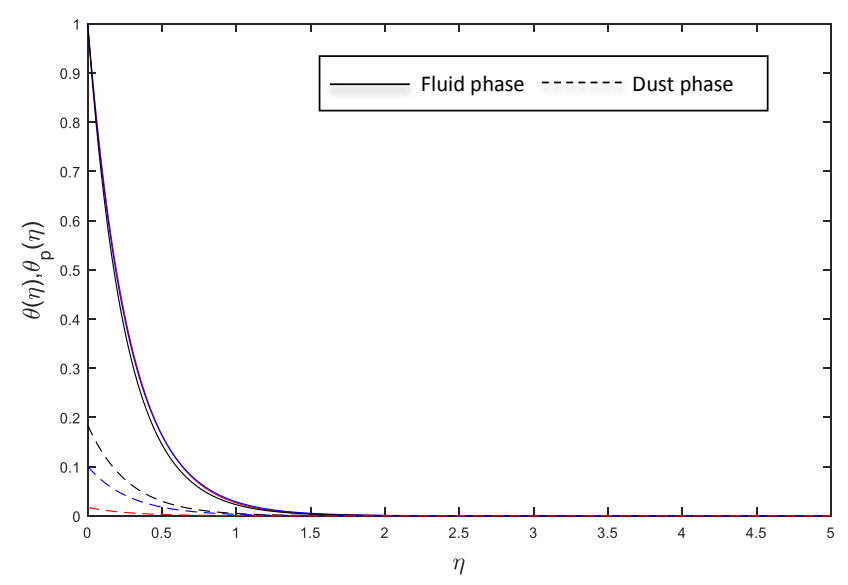

Fig. 5 Temperature profiles for fluid phase $\theta(\eta)$ and dust phase $\theta_{p}(\eta)$ for different values of $\beta$.

Through Figs. 6 and 7, we can see the effect of porosity $k$ on velocity and temperature. Due to the presence of porous medium, we expect that it will increase the resistive force in fluid motion, as a result, we can observe that as $k$ increases, velocity for fluid and dust decreases. However, the temperature for fluid and dust have a slight increase as $k$ increases. This phenomenon occurs due to the thickening thermal boundary layer as porosity increases.

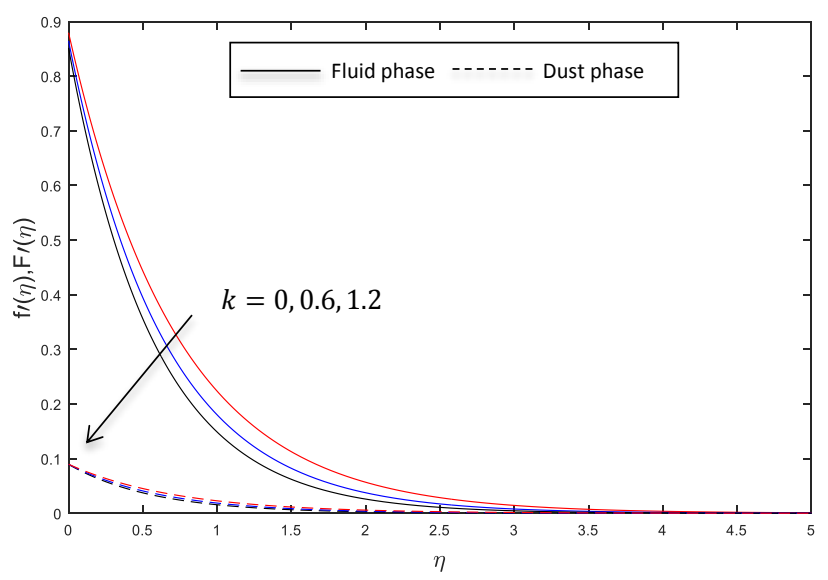

Fig. 6 Velocity profiles for fluid phase $f^{\prime}(\eta)$ and dust phase $F^{\prime}(\eta)$ for different values of $k$.

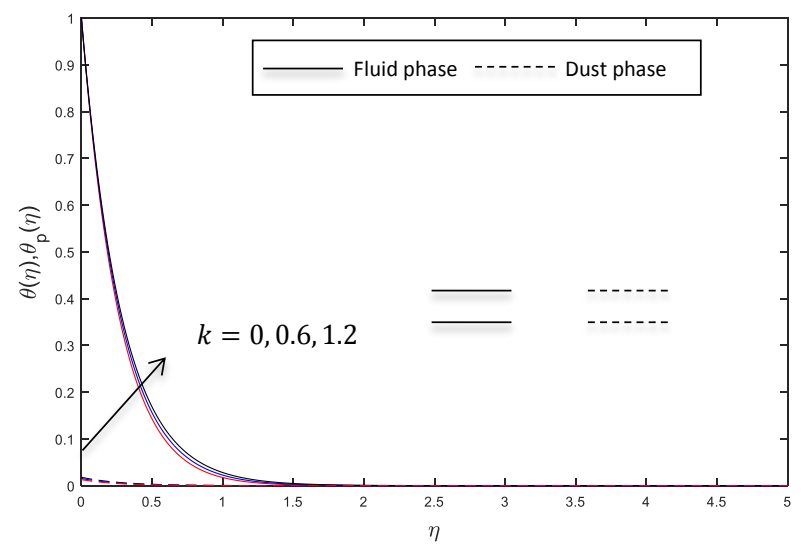

Fig. 7 Temperature profiles for fluid phase $\theta(\eta)$ and dust phase $\theta_{p}(\eta)$ for different values of $k$.

Figs. 8 and 9 show the effect of mass concentration $\rho_{r}$ on velocity and temperature It is obvious from the figure that as $\rho_{r}$ increases, the velocities for fluid and dust decrease. This is because the 
larger mass concentration will slow the movement of fluid and dust. However, the temperature showsonly a slight increase as $\rho_{r}$ increases.

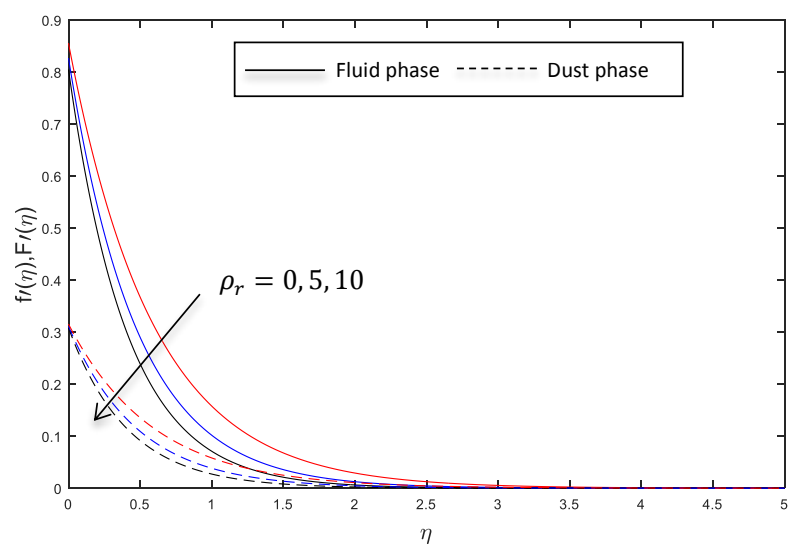

Fig. 8 Velocity profiles for fluid phase $f^{\prime}(\eta)$ and dust phase $F^{\prime}(\eta)$ for different values of $\rho_{r}$.

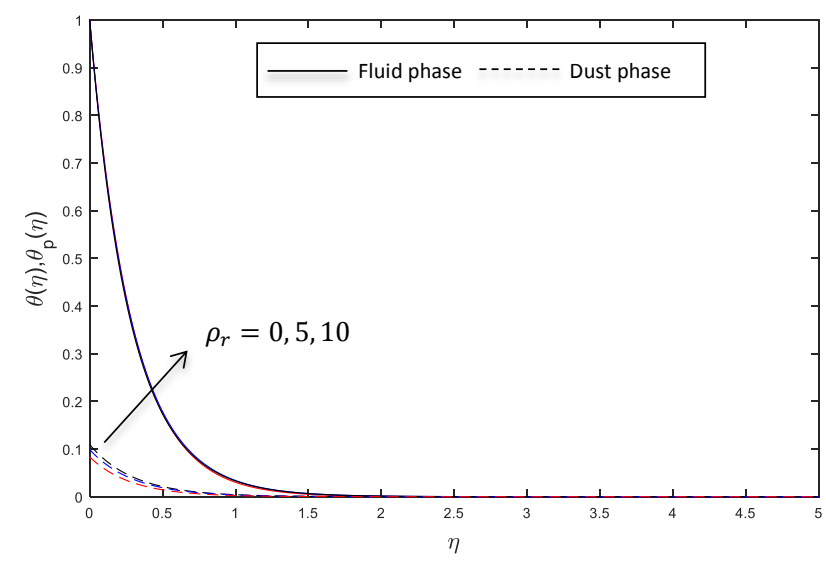

Fig. 9 Temperature profiles for fluid phase $\theta(\eta)$ and dust phase $\theta_{p}(\eta)$ for different values of $\rho_{r}$.

The temperatures for different values of Prandtl number $P r$ are shown in Fig. 10. We observed that the increasing number of Pr implies the decreasing temperature of fluid and dust phase which implies the thicker momentum boundary layer than the thermal boundary layer.

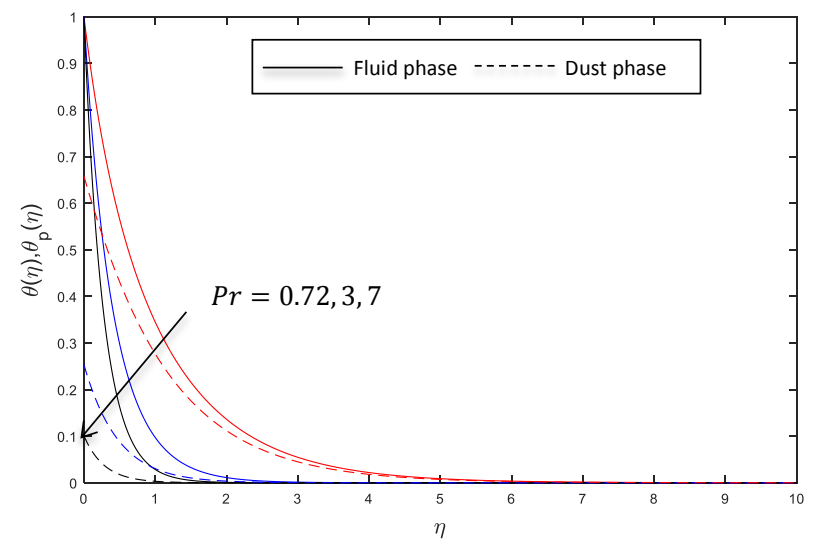

Fig. 10 Temperature profiles for fluid phase $\theta(\eta)$ and dust phase $\theta_{p}(\eta)$ for different values of $\mathrm{Pr}$.

Table 2 displays the velocity gradient $-f^{\prime \prime}(0)$ and temperature gradient $-\theta^{\prime}(0)$. Examining the table reveals that the increasing values of $M, \rho_{r}, k$ and $\beta$ will decrease the skin friction coefficient and the increasing values of $\rho_{r}, \beta$ and $\operatorname{Pr}$ decrease the rate of heat transfer at the surface.

Table 2 Values of velocity gradient $-f^{\prime \prime}(0)$ and wall temperature gradient $-\theta^{\prime}(0)$ for different $M, \beta, \rho_{r}, k$ and $P r$.

\begin{tabular}{|c|c|c|c|c|c|c|}
\hline$M$ & $\rho_{r}$ & $\boldsymbol{\beta}$ & $k$ & $P r$ & $-f^{\prime \prime}(o)$ & $-\theta^{\prime}(0)$ \\
\hline 0 & & & & & 1.8158 & 3.7388 \\
\hline 0.5 & 10 & 0.5 & 1 & 7 & 1.8863 & 3.7010 \\
\hline \multirow[t]{2}{*}{1.0} & & & & & 1.9528 & 3.6651 \\
\hline & 0 & & & & 1.4455 & 3.4716 \\
\hline \multirow[t]{3}{*}{1} & 5 & 0.5 & 1 & 7 & 1.7314 & 3.5503 \\
\hline & 10 & & & & 1.9528 & 3.6651 \\
\hline & & 0.1 & & & 1.6190 & 3.4761 \\
\hline \multirow[t]{3}{*}{1} & 10 & 0.5 & 1 & 7 & 1.9528 & 3.6651 \\
\hline & & 1.0 & & & 2.1153 & 3.9388 \\
\hline & & & 0 & & 1.8158 & 3.7388 \\
\hline \multirow[t]{3}{*}{1} & 10 & 0.5 & 0.5 & 7 & 1.8863 & 3.7010 \\
\hline & & & 1.0 & & 1.9528 & 3.6651 \\
\hline & & & & 0.72 & 1.9528 & 1.2152 \\
\hline \multirow[t]{2}{*}{1} & 10 & 0.5 & 1 & 3 & 1.9528 & 2.5157 \\
\hline & & & & 7 & 1.9528 & 3.6651 \\
\hline
\end{tabular}

\section{CONCLUSION}

The effect of some parameters on hydromagnetic boundary layer flow of a dusty fluid in a porous medium over a stretching sheet with slip effect is investigated. The partial differential equations governing the problem were transformed into a set of nonlinear ordinary differential equations by using similarity transformation. The influence of different parameters including fluid particle interaction parameter $\beta$, magnetic parameter $M$, porosity parameter $k$, Prandtl number $\operatorname{Pr}$ and mass concentration $\rho_{r}$ on velocity, temperature and gradients of both velocity and temperature are shown.. In conclusion,:

- The effects of $M, k$ and $\rho_{r}$ is to decrease the momentum boundary layer thickness.

- The effect of $\beta$ is to increase the momentum boundary layer thickness of fluid. However, it reduces the momentum boundary layer thickness of dust..

- Increasing the Pr decreases the thermal boundary layer thickness.

- Increasing values of $M, \rho_{r}, k$ and $\beta$ decrease the skin friction.

- Increment of $\rho_{r}, \beta$ and $\operatorname{Pr}$ decrease the rate of heat transfer.

\section{ACKNOWLEDGEMENT}

This work was financially supported by the Universiti Tun Hussein Onn Malaysia and Ministry of Higher Education Malaysia.

\section{REFERENCES}

Chakrabarti K. M. 1977. Note on boundary layer in a dusty gas. American Institute of Aeronautics and Astronautics Journal. 12, 136-137.

Crane L. J. 1970. Flow past a stretching plate. Journal of Applied Mathematics and Physics. 21 (4), 645-647.

Fazlina A., Anuar I. Roslinda M. N. 2010, Boundary layer flow and heat transfer adjacent to a stretching vertical sheet with prescribed surface heat flux. MATEMATIKA. 26 (2), 197-206.

Fischer E. G. 1976. Extrusion of Plastics. New York: Wiley.

Gireesha B. J., Ramesh G. K., Lokesh H. J., Bagewadi C. S. 2011. Boundary layer flow and heat transfer of a dusty fluid over a stretching vertical surface. Applied Mathematics. 2, 475-481. 
Grubka L. J., Bobba K. M., 1985. Heat transfer characteristics of a continuous, stretching surface with variable temperature. Journal of Heat Transfer. 107, 248-250.

Mansur S., Ishak A. 2013. The flow and heat transfer of a nanofluid past a stretching/shrinking sheet with a convective boundary condition. Abstract and Applied Analysis. 2013, doi:10.1155/2013/350647. 1-8.

Mansur S., Ishak A., Pop I. 2015. The magnetohydrodynamic stagnation point flow of a nanofluid over a stretching/shrinking sheet with suction. PLoS ONE, 10(3), e0117733, doi:10.1371/journal.pone.0117733.

Sharena M. I., Anati A., Sharidan S. 2016, Magnetohydrodynamic flow of dusty fluid past a vertical stretching sheet with hall effect. Seminar Kebangsaan
Sains Matematik 23 (SKSM23). 24-26 November 2015. Pulai Spring Resort, Johor Bahru. AIP Conference Proceedings 1750, 020005, doi: 10.1063/1.4954518

Vajravelu K., Nayfeh J. 1992. Hydromagnetic flow of a dusty fluid over a stretching sheet. International Journal of Non-Linear Mechanics. 27, 937945.

Vajravelu K., Prasad K. V., Datti P. S. 2013. Hydromagnetic fluid flow and heat transfer at a stretching sheet with fluid-particle suspension and variable fluid properties. Journal of Fluids Engineering. 135, 1-9. 